EPJ manuscript No.

(will be inserted by the editor)

\title{
Anomalous dephasing of bosonic excitons interacting with phonons in the vicinity of the Bose-Einstein condensation
}

\author{
O.M. Schmitt ${ }^{1}$, D.B. Tran Thoai $^{2}$, P. Gartner ${ }^{3}$ and H. Haug ${ }^{1}$ \\ ${ }^{1}$ Institut für Theoretische Physik, J.W.Goethe-Universität Frankfurt, Germany \\ 2 permanent address: Institut of Physics, Ho Chi Minh City, Vietnam \\ ${ }^{3}$ permanent address: National Institut for Physics of Materials, Bucharest, Romania
}

Received: date / Revised version: date

\begin{abstract}
The dephasing and relaxation kinetics of bosonic excitons interacting with a thermal bath of acoustic phonons is studied after coherent pulse excitation. The kinetics of the induced excitonic polarization is calculated within Markovian equations both for subcritical and supercritical excitation with respect to a Bose-Einstein condensation (BEC). For excited densities $n$ below the critical density $n_{c}$, an exponential polarization decay is obtained, which is characterized by a dephasing rate $\Gamma=\frac{1}{T_{2}}$. This dephasing rate due to phonon scattering shows a pronounced exciton-density dependence in the vicinity of the phase transition. It is well described by the power law $\Gamma \propto\left(n-n_{c}\right)^{2}$ that can be understood by linearization of the equations around the equilibrium solution. Above the critical density we get a non-exponential relaxation to the final condensate value $p^{0}$ with $|p(t)|-\left|p^{0}\right| \propto \frac{1}{t}$ that holds for all densities.

Furthermore we include the full self-consistent Hartree-Fock-Bogoliubov (HFB) terms due to the excitonexciton interaction and the kinetics of the anomalous functions $F_{k}=\left\langle a_{k} a_{-k}\right\rangle$. The collision terms are analyzed and an approximation is used which is consistent with the existence of BEC. The inclusion of the coherent $\mathrm{x}$-x interaction does not change the dephasing laws. The anomalous function $F_{k}$ exhibits a clear threshold behaviour at the critical density.
\end{abstract}

PACS. 71.35.Lk Collective effects( Bose effects and excitonic phase transition) - 71.35.-y Excitons 


\section{Introduction}

Bose-Einstein condensation (BEC) is a fascinating topic attracting new interest, due to recent experimental observation for BEC of atomic ensembles [1,2] and evidence for BEC of excitons in semiconductors [3, [4, 5, 6]. In spite of the many similarities between bosonic atoms and excitons in semiconductors with large binding energy, the BEC of the latter has the interesting specific feature that the order parameter of the excitonic condensate is identical to its optical polarization. Therefore it is directly accessible by relatively simple optical measurements. This fact stimulated theoretical predictions of unusual nonlinear optical properties in the condensed regime [7].

In this paper the decay of the polarization $p$ (=order parameter) is studied after excitation with a coherent laser beam, if one approaches the critical density $n_{c}$ for a BEC, where an unusual dephasing kinetics can be expected. Unlike most studies of the condensation kinetics [8, 9, 10], here a large macroscopic occupation of the condensate is created by the excitation process via allowed optical transitions. This induced exciton amplitude may then decay to its zero or nonzero stationary value. The final stationary solution is perfectly described by the equilibrium theory of the free Bose gas.

One of the most promising candidates for an excitonic BEC is $\mathrm{Cu}_{2} \mathrm{O}$ 11] with its extremely stable excitons. Due to its dipole forbidden exciton transition, an experiment corresponding to our simulations would use two-photon transitions for the excitation of ortho-excitons and a timeresolved polarization measurement via the same mecha- nism [13]. Although for a detailed quantitative simulation of such an experiment, additional effects like polariton effects [12], lifetime effects [10] and the subtle excitation mechanism will play a role even in the low density limit, our main predictions should be observable at least qualitatively also in $\mathrm{Cu}_{2} \mathrm{O}$ close to a $\mathrm{BEC}$.

\section{Incoherent relaxation and dephasing}

\section{kinetics for excitons interacting with a bath of acoustic phonons}

1s-excitons with center-of-mass momentum $\mathbf{k}$ are treated in the boson approximation, i.e. the exciton operators fulfill the commutation relation $\left[a_{\mathbf{k}}, a_{\mathbf{k}^{\prime}}^{+}\right]_{-}=\delta_{\mathbf{k}, \mathbf{k}^{\prime}}$. This approximation is only justified in semiconductors with large exciton binding energy, when $n a_{0}^{3} \ll 1$ and mainly 1 sexcitons are excited. These excitons interact with longitudinal acoustic phonons and a coherent classical light pulse via a dipole interaction. The Hamiltonian is given by

$$
\begin{aligned}
& H=\sum_{\mathbf{k}} e_{\mathbf{k}} a_{\mathbf{k}}^{\dagger} a_{\mathbf{k}}+\sum_{\mathbf{q}} \hbar \omega_{\mathbf{q}} b_{\mathbf{q}}^{\dagger} b_{\mathbf{q}} \\
& +\frac{1}{\sqrt{V}} \sum_{\mathbf{k}, \mathbf{q}} g_{\mathbf{q}} a_{\mathbf{k}+\mathbf{q}}^{\dagger} a_{\mathbf{k}}\left(b_{\mathbf{q}}+b_{-\mathbf{q}}^{\dagger}\right)-\sqrt{V}\left(d E(t) a_{0}^{\dagger}+h . c .\right)
\end{aligned}
$$

with the dispersion of the excitons and phonons

$$
e_{\mathbf{k}}=\frac{\hbar^{2} \mathbf{k}^{2}}{2 m} \quad, \quad \omega_{\mathbf{q}}=c|\mathbf{q}|
$$

respectively, where $m$ is the translational exciton mass and $c$ the sound velocity. The deformation potential matrix element

$$
g_{\mathbf{q}}=G \sqrt{\hbar \omega_{\mathbf{q}}}
$$

in the long-wave length limit. The interaction constant $G$ is given in terms of the deformation potential $D$ and the 
crystal density $\rho$ by $G^{2}=\frac{D^{2}}{\rho c^{2}}$. The finite photon momentum has been neglected.

We define further the exciton distribution function $n_{k}=$ $\left\langle a_{k}^{\dagger} a_{k}\right\rangle$ with $k \neq 0$ and the exciton polarization amplitude $p=\frac{1}{\sqrt{V}}\left\langle a_{0}\right\rangle$.

One gets a closed set of kinetic equations for the polarization $p$ and the exciton occupation $n_{k}$, by extending the standard method for deriving a semiclassical Boltzmann equations also to the order parameter $p$ [8]. The Heisenberg equations for $\left\langle a_{0}\right\rangle$ and $\left\langle a_{k}^{+} a_{k}\right\rangle$ are iterated to second order in the interaction potential $g_{q}$. The higher order mean-values are factorized and the only correlations kept are $p$ and $n_{k}$, e.g.

$$
\left\langle a a^{+} a b^{+} b\right\rangle \approx\left(1+\left\langle a^{+} a\right\rangle\right)\langle a\rangle\left\langle b^{+} b\right\rangle+\langle a\rangle\left\langle a^{+} a\right\rangle\left\langle b^{+} b\right\rangle .
$$

Neglecting principal value contributions as well as finite lifetime effects and performing the Markov limit, one arrives at 8

$$
\begin{aligned}
\frac{\partial}{\partial t} n_{\mathbf{k}} & =-\frac{1}{V} \sum_{\mathbf{k}^{\prime}}\left\{W_{\mathbf{k k}^{\prime}} n_{\mathbf{k}}\left(1+n_{\mathbf{k}^{\prime}}\right)-\left(\mathbf{k} \rightleftharpoons \mathbf{k}^{\prime}\right)\right\} \\
& -\left[W_{\mathbf{k} 0} n_{\mathbf{k}}-W_{0 \mathbf{k}}\left(1+n_{\mathbf{k}}\right)\right]|p|^{2} \\
\frac{\partial}{\partial t} p & =\frac{1}{2 V} \sum_{\mathbf{k}^{\prime}}\left[W_{\mathbf{k}^{\prime} 0} n_{\mathbf{k}^{\prime}}-W_{0 \mathbf{k}^{\prime}}\left(1+n_{\mathbf{k}^{\prime}}\right)\right] p \\
& +\frac{i}{2 \hbar} d E_{0}(t) .
\end{aligned}
$$

The transition rates are given by Fermi's golden rule

$$
\begin{aligned}
W_{\mathbf{k k}^{\prime}} & =\frac{2 \pi G^{2}}{\hbar}\left|e_{\mathbf{k}}-e_{\mathbf{k}^{\prime}}\right|\left[N_{\mathbf{k}^{\prime}-\mathbf{k}} \delta\left(e_{\mathbf{k}}-e_{\mathbf{k}^{\prime}}+\hbar \omega_{\mathbf{k}^{\prime}-\mathbf{k}}\right)\right. \\
& \left.+\left(1+N_{\mathbf{k}-\mathbf{k}^{\prime}}\right) \delta\left(e_{\mathbf{k}}-e_{\mathbf{k}^{\prime}}-\hbar \omega_{\mathbf{k}-\mathbf{k}^{\prime}}\right)\right]
\end{aligned}
$$

where $N_{k}=\frac{1}{e^{\beta \hbar \omega_{k}-1}}$ is the thermal distribution of the phonons. The delta-functions are broadened into Lorentz -ians according to 8 . In what follows (except in the last chapter) we will study the consequence of these equations.

\section{Laser pulse induced Bose Einstein} condensation

The strength of the laser pulse can be varied to excite densities below (see Fig.11) and above (see Fig. 21) the critical density for the BEC. The laser pulse is tuned to the lowest 1s-exciton resonance and has a duration of $2.5 \mathrm{~ns}$. As an example $Z n S e$ parameters have been chosen at a low temperature of $0.5 K$. Although the BEC in $Z n S e$ may be unlikely, this semiconductor with its dipole allowed excitons serves well to demonstrate our ideas, which may hold for different experimental setups and materials.

In Fig. 11 and Fig. 2 a light pulse induced condensation is obtained. At excitation densities below the critical density the polarization $|p|$ decays exponentially until it vanishes as expected from usual optical experiments (Fig. 11). At stronger excitations above the critical density, the exciton polarization approaches non-exponentially its finite stationary value (Fig. 2), which is non-zero due to the onset of condensation. This stationary polarization is given in terms of the excited density $n$ and the temperature $T$ in the form of $\left|p^{0}\right|=\sqrt{n-n_{c}(T)}$ as expected form the equilibrium theory of the Bose gas. Obviously the finite lifetime of the excitons due to spontaneous recombination will eventually cause an additional dephasing and a decay of the exciton density. 


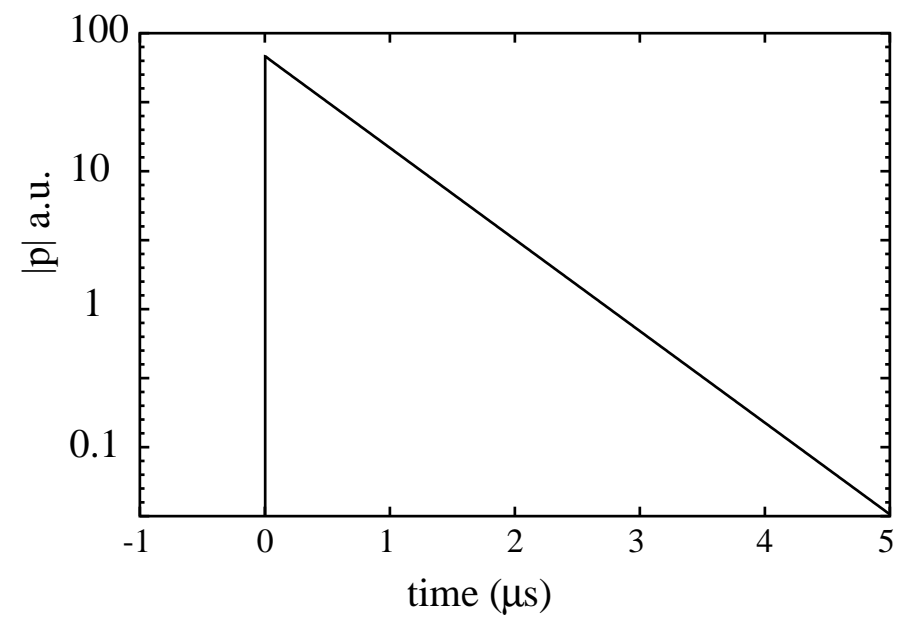

Fig. 1. Complete exponential dephasing for excitation with $n<n_{c}$

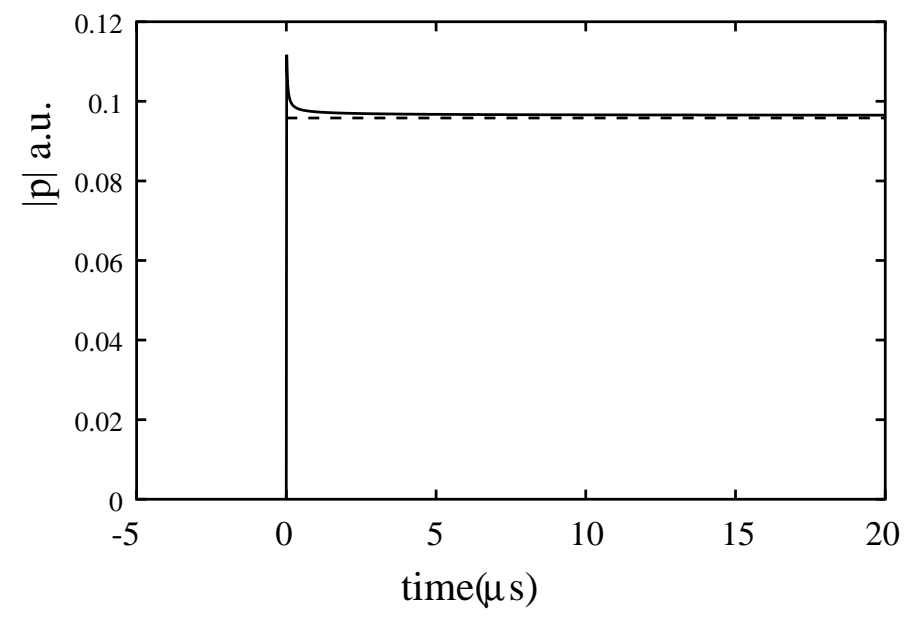

Fig. 2. Non-exponential approach of the exciton amplitude to condensation for excitations with $n>n_{c}$. Full line - calculated polarization, dashed line - value predicted from equilibrium theory of the Bose-gas

\section{Critical slowing-down of the dephasing for} approaching the critical density from below

Below the critical density the decay of the polarization is exponential, therefore a dephasing time $T_{2}$ and a dephasing rate $\Gamma=\frac{1}{T_{2}}$ can be defined. We study the excitondensity dependence of these quantities as one approaches the critical density from below. It is generally assumed that the contribution of the phonons to the dephasing rate is approximately density-independent. We can reproduce this result for moderate densities but do get a considerable reduction of the dephasing rate near the critical density as shown in Fig. 3. The density dependence of the dephasing rate can be fitted extremely well by a quadratic law

$$
\Gamma \propto\left(n-n_{c}\right)^{2}
$$

which vanishes at the critical density, as also shown in Fig. 3. This characteristic slowing-down of the exciton-

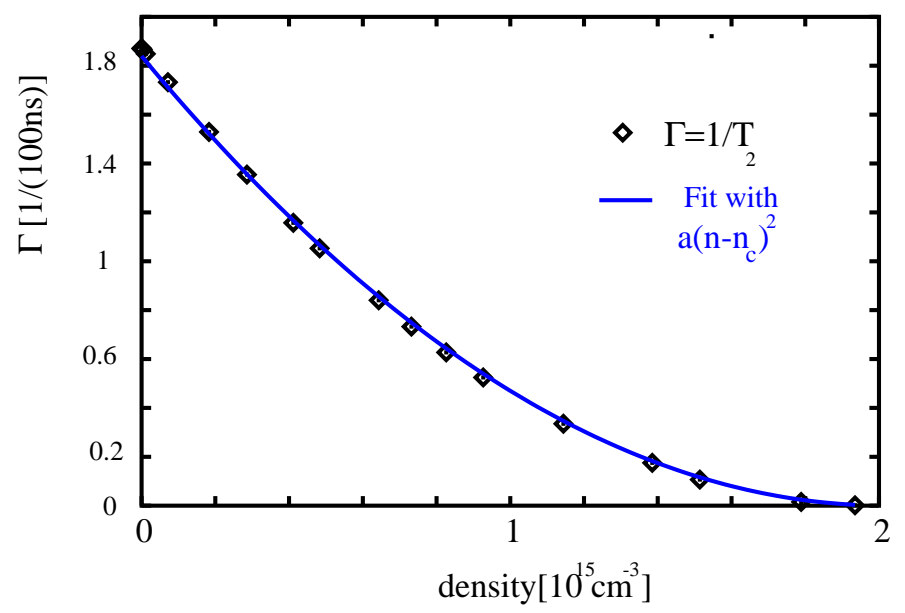

Fig. 3. Density dependence of the dephasing rate $\Gamma$ for $n_{c}=$ $2 \cdot 10^{15} \mathrm{~cm}^{-3}$.

dephasing kinetics can serve as a first experimental sign for the approach to the BEC.

A similar result has been observed also for exciton-exciton scattering within similar rate equations 14 .

The quadratic law Eq.(7) can be derived analytically by linearizing Eqs. 4 and 5 around the stationary solutions $\left|p^{0}\right|$ and $n_{k}^{0}$. For the deviation $\delta p$ from the equilibrium 
value $p^{0}=0$ one gets:

$$
\frac{\partial}{\partial t} \delta p=\frac{1}{2 V} \sum_{\mathbf{k}^{\prime}}\left[W_{\mathbf{k}^{\prime} 0} n_{\mathbf{k}^{\prime}}^{0}-W_{0 \mathbf{k}^{\prime}}\left(1+n_{\mathbf{k}^{\prime}}^{0}\right)\right] \delta p
$$

Therefore one can immediately conclude that the final decay for $n<n_{c}$ is exponential and furthermore

$$
\Gamma=\frac{1}{T_{2}}=\frac{1}{2 V}\left(1-e^{\beta \mu}\right) \sum_{\mathbf{k}} W_{\mathbf{k} 0} \frac{1}{e^{\beta e_{k}}-e^{\beta \mu}} .
$$

On the other hand $\left(1-e^{\beta \mu}\right) \propto\left(n-n_{c}\right)^{2}$ holds as an exact thermodynamic relationship between the leading terms for $\mu \approx 0$ and $n \approx n_{c}$. However, the relation is relatively well obeyed even for large departures from the critical values, as can be checked by a simple numerical calculation Furthermore, due to the structure of the transition rates Eq.(6), the main contribution in the sum of Eq.(9) stems from the $k$-values away from the origin (the nonzero solution of the delta-function). Therefore, for small temperatures the $\mu$-dependence of the sum is weak and does not affect the $\left(n-n_{c}\right)^{2}$ behaviour of its prefactor.

\section{Power law relaxation to the equilibrium}

\section{condensate for $n>n_{c}$}

While the polarization $|p|$ vanishes exponentially at subcritical excitation, it reaches its stationary value $\left|p^{0}\right|=$ $\sqrt{n-n_{c}} \neq 0$, when the excited density $n$ exceeds $n_{c}$. But in contrast to the subcritical behaviour, this approach to equilibrium of the ideal Bose gas is not exponential any more. In contrast the dephasing kinetics is well described by a simple power law,

$$
|p(t)|=\left|p^{0}\right|+a /(t+b),
$$

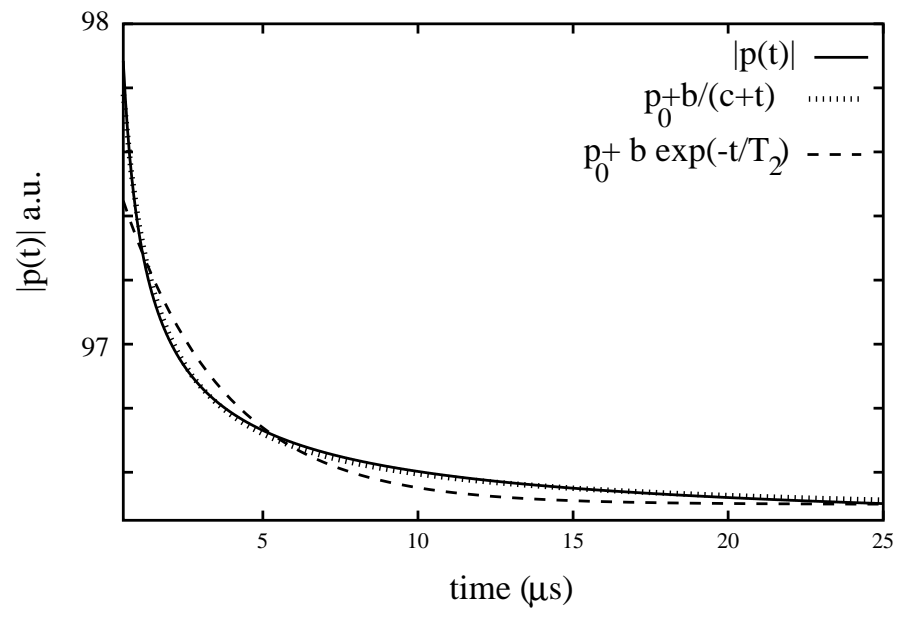

Fig. 4. Asymptotic kinetics of $|p(t)|$ for excitations with $n>$ $n_{c}$, power law fit $|\delta p(t)| \propto \frac{1}{t+c}$ dotted line. Exponential fit dashed line.

as shown in Fig. 4 .

The slow non-exponential approach to equilibrium for $n>n_{c}$ would also give in experiments sensitive to coherence a clear signature for the BEC, which may be observable even when the final condensate is small and cannot be detected clearly.

Above the critical density the same linearization procedure like the one used in the last section but with $\mu=0$, $p=p^{0}+\delta p$ and $p^{0} \neq 0$ yields

$$
\frac{\partial}{\partial t} \delta p=\frac{1}{2 V} \sum_{\mathbf{k}}\left(1-e^{-\beta e_{k}}\right) W_{k 0} \delta n_{k} p^{0}
$$

As shown in Ref. [8], the long-time behaviour for $\delta n_{k}$ is not exponential. The spectrum of the linear operator controlling the $t \rightarrow \infty$ asymptotics of $\delta n_{k}$ is continuous and starts from zero. Therefore, it is expected that a power-law decay is obeyed, which is in accordance with our numerical results. 


\section{Combination of phonon-scattering with HFB-correlations}

The model used in the last sections may be useful to describe the incoherent dephasing and relaxation phenomena of the light-induced BEC. However, it does not account for a variety of interesting and important phenomena, where exciton-exciton correlations play an important role. These excitonic correlations are known to be important for the nonlinear optical response of semiconductors 15, 16, 17. Additionally, essential aspects of the BEC can be only understood in terms of an interacting Bose-gas. These phenomena are e.g. superfluidity [3, 4], the renormalized spectrum of the elementary excitations and some interference phenomena based on the GrossPitajevski equation. These effects are taken into account by the self-consistent Hartree-Fock-Bogoliubov treatment of the boson-boson interaction that is also called GirardeauArnowitt approximation. The impact of this approximation on the BEC has been reviewed e.g. in Refs. [18]. We will now give a guideline how to include the HFB approximation in the phonon scattering kinetics in such a way, that the results of the last sections are conserved, but moreover coherent aspects are taken into account.

In the following the derivation of these equations is described.

To the Hamiltonian Eq. (11) the exciton-exciton interaction $H^{\prime}$ with a contact potential $W$ is added

$$
H^{\prime}=\frac{1}{4 V} \sum_{\mathbf{k}_{1}, \mathbf{k}_{2}, \mathbf{q}} W a_{\mathbf{k}_{1}}^{+} a_{\mathbf{k}_{2}}^{+} a_{\mathbf{k}_{1}+\mathbf{q}} a_{\mathbf{k}_{2}-\mathbf{q}}
$$

The interaction matrix element $W$ between the excitons is in general given by a momentum-dependent expectation value of the Coulomb interactions between the various point charges in the two excitons containing both the attractive direct and the repulsive exchange interaction. In a low-temperature exciton system the small momentum transfer dominates. In this limit the direct interaction can be neglected and the exchange integral can be calculated analytically [19]. This yields a repulsive contact potential with the coupling constant

$$
W=\frac{26}{3} \pi a_{0}^{3} E_{r}
$$

Here $a_{0}$ and $E_{r}$ are the Bohr radius and the Rydberg energy, respectively. Note however, that the possibility of a biexciton formation is lost in this long-wavelength approximation.

In the HFB approximation all possible contractions have to be taken which conserve the one-particle structure of the Hamiltonian and its translational invariance. Therefore we have to introduce additionally to $p$ and $n_{k}$ the anomalous function $F_{k}(t)=\left\langle a_{k}(t) a_{-k}(t)\right\rangle$. From the viewpoint of the underlying e-h picture, the bosonic HFB approximation includes also the dynamics of four-particle correlations (two electron and two hole operators). Therefore, correlations beyond the usual electronic HF approximation are taken into account.

To derive the kinetics, the Heisenberg equations are iterated to first order in $W$ and to second order in $g$. The mean-values of higher orders are then factorized as explained in section 2, only that now all possible con- 
tractions are taken into account including the anomalous function $F_{k}$. The phonon part(collision terms) is treated in the Markov approximation and contributions from principal value integrals have been neglected. Due to the fact that the number of possible second-order phonon contributions is quite large, all anomalous functions $F_{k}$ are neglected in the collision terms. We will show later that the contributions of the anomalous functions to the collision terms within the same approximation scheme make the condensate unstable. In the language of diagram theory, our approximation includes the HFB diagrams [14] and a selection of second-order phonon scattering diagrams.

The resulting equations are

$$
\begin{aligned}
& \frac{\partial}{\partial t} n_{k}=\frac{W}{\hbar} \Im\left\{\left(p^{2}+\frac{1}{V} \sum_{\mathbf{q}} F_{q}\right) F_{k}^{*}\right\} \\
& +\left.\frac{\partial}{\partial t} n_{k}\right|_{c o l l} \\
& \frac{\partial}{\partial t} F_{k}=-\frac{i}{\hbar}\left[2 e_{k}+2 W\left(n_{0}+\frac{1}{V} \sum_{\mathbf{q}} n_{q}\right)\right] F_{k} \\
& -\frac{i}{2 \hbar}\left(2 n_{k}+1\right) W\left(p^{2}+\frac{1}{V} \sum_{\mathbf{q}} F_{q}\right)+\left.\frac{\partial}{\partial t} F_{k}\right|_{c o l l} \\
& \frac{\partial}{\partial t} p=-\frac{i}{\hbar}\left[e_{0}+\frac{W}{2}\left(n_{0}+\frac{2}{V} \sum_{\mathbf{q}} n_{q}\right)\right] p \\
& -\frac{i}{2 \hbar} W p^{*} \frac{1}{V} \sum_{\mathbf{q}} F_{q}+\frac{i}{2 \hbar} d E+\left.\frac{\partial}{\partial t} p\right|_{\text {coll }} \\
& \left.\frac{\partial}{\partial t} n_{k}\right|_{\text {coll }}=-\frac{2 \pi}{\hbar V} \sum_{\mathbf{q}} g_{\mathbf{k}-\mathbf{q}}^{2}\left\{\delta\left(e_{q}-e_{k}-\hbar \omega_{\mathbf{k}-\mathbf{q}}\right)\right. \\
& \left.\times\left[N_{k-q} n_{k}\left(1+n_{q}\right)-\left(N_{k-q}+1\right)\left(1+n_{k}\right) n_{q}\right]-(\mathbf{k} \leftrightarrow \mathbf{q})\right\} \\
& +\frac{2 \pi}{\hbar} g_{k}^{2} \delta\left(e_{k}-\hbar \omega_{k}\right) n_{0}\left(N_{k}-n_{k}\right) \\
& \left.\frac{\partial}{\partial t} p\right|_{\text {coll }}=-\frac{\pi}{\hbar V} p \sum_{\mathbf{q}} g_{q}^{2} \delta\left(e_{q}-\hbar \omega_{q}\right)\left(N_{q}-n_{q}\right) \\
& \left.\frac{\partial}{\partial t} F_{k}\right|_{c o l l}=-\frac{2 \pi}{\hbar} g_{k}^{2} \delta\left(e_{k}-\hbar \omega_{k}\right)\left[p^{2}\left(N_{k}-n_{k}\right)\right]
\end{aligned}
$$

First we demonstrate that the addition of scattering terms proportional to $F_{k}$ in the collision terms of $F_{k}$ Eq. (19) destroy the condensate, while omitting them leads to a stable condensate solution. In Fig. 5 a solution of the equations is shown above the critical density. While the coherent density $n_{0}=|p|^{2}$ goes to zero if the collision terms proportional to $F_{k}\left(F^{1}\right.$-terms) are included (see curves 3,4 ), it condenses if only terms without $F_{k}\left(F^{0}\right.$-terms $)$ are considered as done in Eq.(19) (see curves 1,2). For subcritical

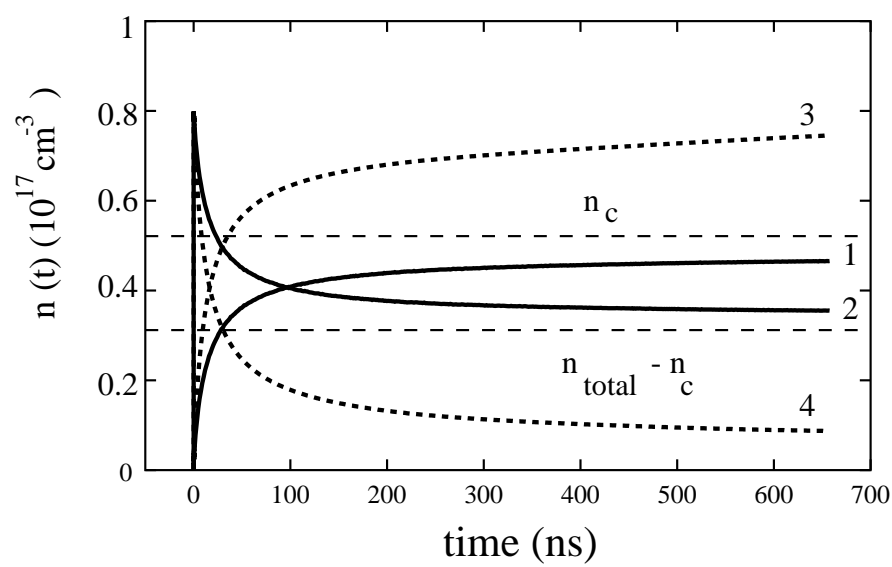

Fig. 5. Density $n$ and $n_{0}$ versus time $t$ above the critical density with $n_{\text {total }}=8.3 \cdot 10^{16} \mathrm{~cm}^{-3}$ and $T_{p h}=1.5 \mathrm{~K}$. Curve 1 and 2: $F$ neglected $\left(F^{0}\right)$ in collision terms. Curve 3 and 4: first order contributions $\left(F^{1}\right)$ included in the collision terms. Horizontal lines are $n_{c}=5.2 \cdot 10^{16}$ and $n_{\text {total }}-n_{c}=3.1 \cdot 10^{16} \mathrm{~cm}^{-3}$ respectively.

excitation the condensate decays in both approximations.

The reason for the numerically obtained condensate destroying properties of the anomalous contributions in the collision terms are not yet fully understood. It can be easily seen analytically that these terms are not in ac- 
O.M. Schmitt et al.: Dephasing of excitons in the vicinity of $n_{c}$

cordance with the condensate solutions of the free Bosegas. However they are obviously also not yet fully compatible with the condensation-solution of the interacting Bose-gas. More refined approximations like the inclusion of energy renormalizations in the collision integrals are needed. An argument for omitting the contributions of the anomalous functions to the second order scattering terms has been proposed in Ref. [20]. Here it is argued that an adiabatic elimination of $F_{k}$ yields for a scattering term containing one anomalous function a term of third-order $\propto W g_{q}^{2}$, which is inconsistent with the considered secondorder scattering. Although this argument is not rigorous in a self-consistent theory, all collision terms proportional to $F_{k}$ are disregarded in the following.

We compare the prediction for the kinetics of the pure phonon model Eqs.(㺼),(5) and the full model Eqs.(14.19) in two figures once below (Fig. 6) and once above (Fig. 17) the critical density.

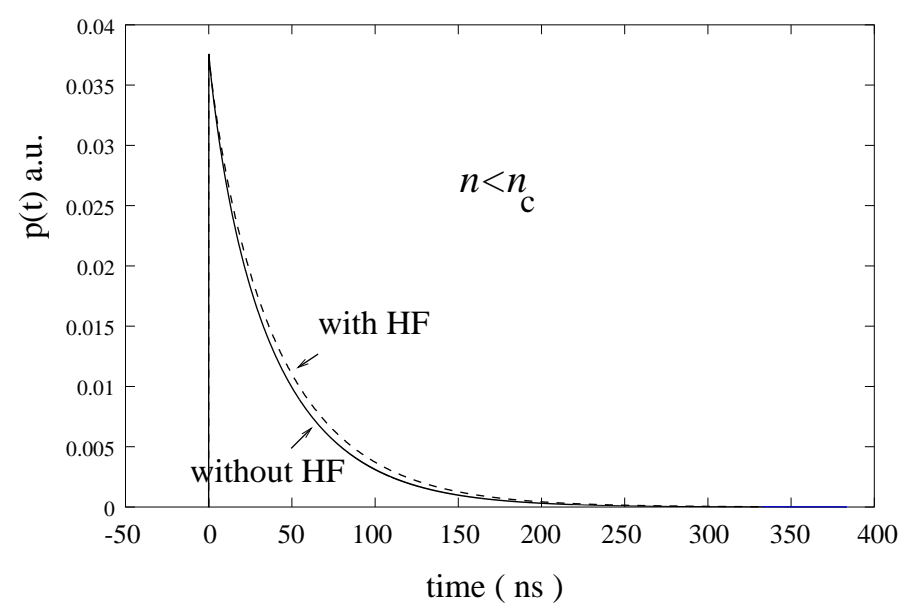

Fig. 6. Excitation at $n<n_{c}$, comparison $\mathrm{HF}$, without $\mathrm{HF}$

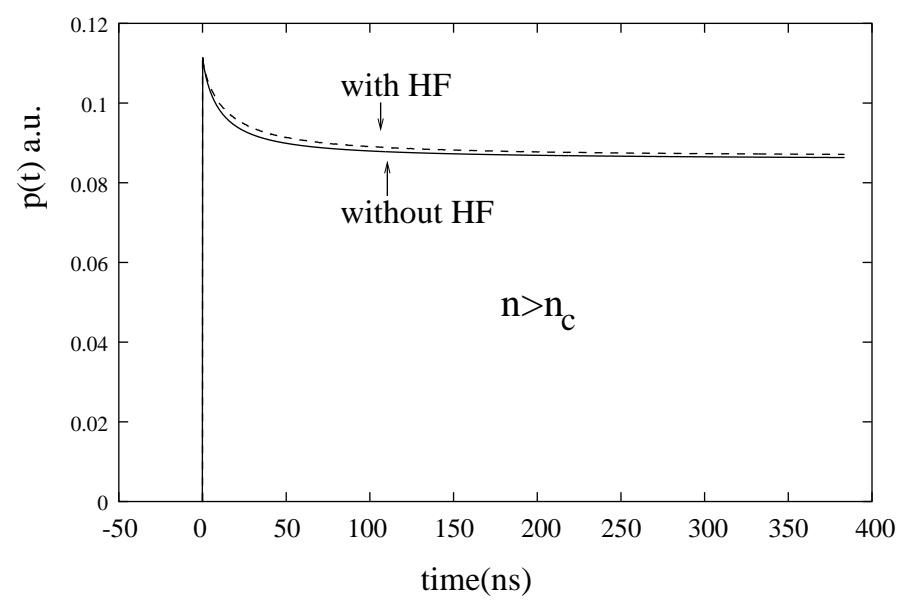

Fig. 7. Excitation at $n>n_{c}$, comparison $\mathrm{HF}$, without HF

The results of the two models concerning dephasing and relaxation can hardly be distinguished. We conclude that all our results in the pure phonon model are stable against Hartree-Fock-Bogoliubov correlations.

Additionally the introduced anomalous function $F_{k}$ shows a threshold behaviour as expected in a phase transition. This is shown in Fig. 8 where we plotted $\sum_{k}\left|F_{k}(t=\infty)\right|$ versus the excitation density.

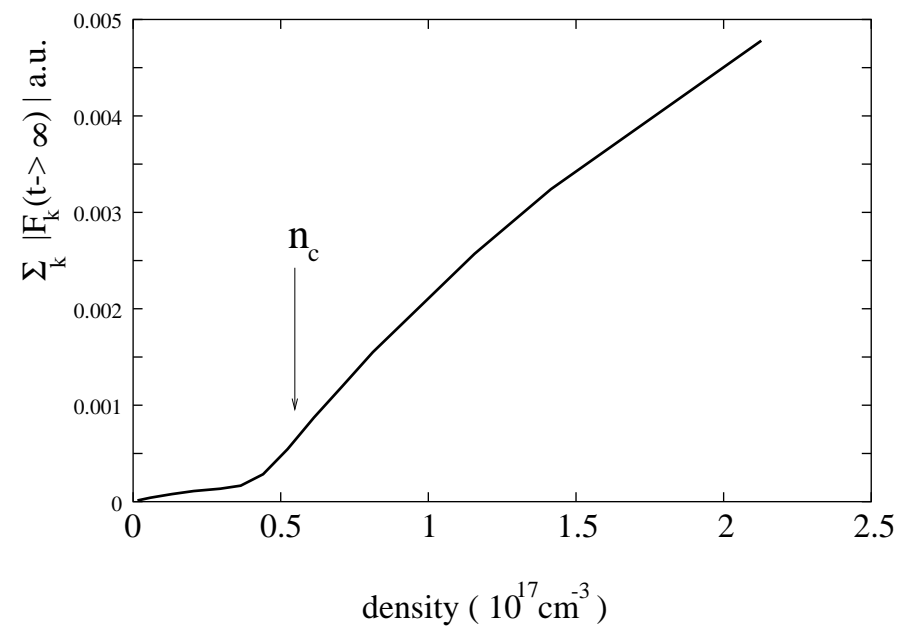

Fig. 8. Asymptotic sum of the anomalous function $F_{k}$

To conclude this chapter, we presented a model which does show the well-understood dephasing and condensa- 
tion properties of the earlier introduced simpler boson model, but additionally can be applied to experiments where the coherence plays a more important role than in simple studies of the dephasing.

\section{Conclusion}

It was demonstrated that the dephasing of an exciton polarization due to phonon scattering can have surprising features, when the BEC is approached. For subcritical excitation with a laser pulse, the polarization dephasing rate slows down with increasing density in the vicinity of the BEC with a quadratic power law. For supercritical excitation the expected exponential dephasing changes to a power law relaxation approaching a finite value. We hope that these results may stimulate corresponding experimental investigations. In the last chapter it was shown that condensation kinetics is very sensitive to the choice of specific scattering terms (diagrams). A model is constructed which includes both the incoherent scattering due to phonons and the HFB correlations. It has the same incoherent properties as the pure phonon model, but may be also applied to experiments, where coherent correlations are important.

This work has been supported by the DFG in the framework of the Schwerpunktprogramm Quantenkohärenz in Halbleitern.

\section{References}

1. M.H. Anderson, J.R. Ensher, M.R. Matthews, C.E. Wiemann, E.A. Cornell, Science 269, 198 (1995)
2. K.B. Davis, M.O. Mewes, M.R. Andrews, N.J. van Druten, D.S. Durfee, D.M. Kurn, W. Ketterle, Phys. Rev. Lett. 75, 3969 (1995)

3. A. Mysyrowicz, E. Benson, and E. Fortin, Phys. Rev. Lett. 77, $896(1996)$

4. L. V. Butov and A. I. Filin, Phys. Rev. B 58, 1980 (1998)

5. J.L. Lin and J.P. Wolfe, Phys. Rev. Lett. 71, 1222 (1993)

6. T. Goto, M. Y. Shen, S. Koyama, and T. Yokuchi, Phys. Rev. B 55, 7609 (1997)

7. Th. Östreich, T. Portengen and L. J. Sham, Solid State Comm. 100, 325 (1996)

8. L. Bányai, P. Gartner, O.M. Schmitt and H. Haug, Condensation kinetics for Bosonic Excitons Interacting with a Thermal Bath of Phonons, Phys. Rev. B, 1. April (2000)

9. A.L. Ivanov, C. Ell, and H. Haug, Phys. Rev. E 55, 6363 (1997)

10. S.G. Tikhodeev, Sov. Phys. JETP 70, 380 (1990)

11. For a review of this semiconductor in respect to BEC, see J.P. Wolfe, J.L. Lin and D.W.Snoke in A. Griffin, D.W. Snoke and S. Stringari, eds., Bose-Einstein Condensation, Cambridge University Press, Cambridge, 1995, p.281

12. C. Ell. A.L. Ivanov and H. Haug, Phys. Rev. B 57, 9663 (1998)

13. D. Fröhlich, K. Reimann and R. Wille, Europhys. Lett. 3, $853(1987)$

14. O.M. Schmitt, L. Bányai and H. Haug, Phys. Rev. B 60, 16506 (1999)

15. V.M. Axt, K. Victor and T. Kuhn, phys. stat. sol.(b) 206, 189 (1998)

16. C. Sieh, T. Meier, A. Knorr, F. Jahnke, P. Thomas and S.W. Koch, Eur. Phys. J. B 11, 407 (1999) 
17. Th. Östreich and L.J. Sham, Phys. Rev. Lett. 83, 3510 (1999)

18. P.C. Hohenberg and P.C. Martin, Annals of Physics 34, $291(1965)$

19. H. Hanamura and H. Haug, Phys. Reports 33C, 209 (1977)

20. N.P. Proukakis, K. Burnett and H.T.C. Stoof, Phys. Rev. A 57, $1230(1998)$ 